\title{
Stationary States of Quantum Dynamical Semigroups *
}

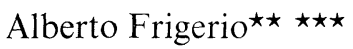 \\ Istituto di Fisica dell'Università, Milano, Italy \\ Istituto Nazionale di Fisica Nucleare, Sezione di Milano, Milano, Italy
}

\begin{abstract}
We study the class of stationary states and the domain of attraction of each of them, for a dynamical semigroup possessing a faithful normal stationary state. We give applications to the approach to stationarity of an open quantum system, and to models of the quantum measurement process.
\end{abstract}

\section{Introduction}

Quantum dynamical semigroups provide a convenient mathematical framework for the study of approach to equilibrium of an open quantum system. In some recent works of several authors, conditions have been found for a dynamical semigroup to possess a unique stationary state and to induce approach to it, in the cases of $N$-level systems $[1,2]$ and of dynamical semigroups with a faithful normal stationary state $[3,4]$; the related problem of irreducibility has been treated in $[5-7]$.

In this note we extend the results of [3] in several respects. Under the only assumptions that the dynamical semigroup $T_{t}$ under consideration acts on a von Neumann algebra $\mathscr{M}$ and possesses (at least) a faithful normal stationary state, which imply, by [3], that the fixed point set of $T_{t}$ in $\mathscr{M}$ is a von Neumann subalgebra of $\mathscr{M}$, we give in Section 2 a classification of the normal stationary states of $T_{t}$. Under an additional condition of sufficient dissipativity, which is less restrictive than the ones of [3], we prove in Section 3 that any normal state tends to a limit as $t \rightarrow \infty$, under the action of $T_{t}$, and we characterize the domain of attraction of each normal stationary state. The results apply to the discussion of approach to stationarity for a spatially confined quantum system weakly coupled to several heat reservoirs at different nonzero temperatures [8] and to some models of the quantum measurement process (cf. [9]). For a restricted class of dynamical semigroups, we prove in Section 4 a stronger property of approach to equilibrium, which should be of interest in the study of infinitely extended quantum systems,

\footnotetext{
* Supported in part by NATO Research Grant No. 1380 and by CNR Research Contract No. 77.01543.63

${ }^{\star \star}$ A fellowship from the Italian Ministry of Public Education is acknowledged

$\star \star \star$ Postal address: Istituto di Scienze Fisiche dell'Università, Via Celoria 16, I-20133 Milano, Italy
} 
in order to discriminate real dissipation of local disturbances from their migration to infinity (cf. [10]).

\section{Classification of Stationary States}

We recall some definitions and preliminaries.

A dynamical semigroup $[11-13,6]$ of a von Neumann algebra $\mathscr{M}$ is a weakly* continuous one-parameter semigroup $\left\{T_{t}: t \geqq 0\right\}$ of completely positive identity preserving normal maps of $\mathscr{M}$ into itself, with $T_{0}$ the identity map $I$. Assume that $T_{t}$ possesses a faithful normal stationary state $\omega$. Then $\mathscr{M}$ can be identified with its representation $\pi_{\omega}(\mathscr{M})$ on the Hilbert space $\mathscr{H}=\mathscr{H}_{\omega}$, with cyclic and separating vector $\Omega$. There exists $[11,14]$ a strongly continuous contraction semigroup $\hat{T}_{t}$ on $\mathscr{H}$ such that $\hat{T}_{t}(A \Omega)=T_{t}(A) \Omega$ for all $A$ in $\mathscr{M}, t \geqq 0$. The set $\mathscr{V}$ of vector functionals, defined by

$$
\mathscr{V}=\left\{\psi \in \mathscr{M}_{*} ; \psi(A)=(\Psi, A \Omega), \Psi \in \mathscr{H}, \forall A \in \mathscr{M}\right\}
$$

is uniformly dense in $\mathscr{M}_{*}$ by [15] Example 5 (see also [10], Theorem 4.2). The fixed point set of $T_{t}$ in $\mathscr{M}$ is a von Neumann subalgebra of $\mathscr{M}$, which we denote by $\mathscr{M}(T)([3]$, Lemma 3 ; using [7], Theorem 3.1).

Theorem 2.1. Let $T_{t}$ be a dynamical semigroup of a von Neumann algebra $\mathscr{M}$, with a faithful normal stationary state $\omega$. Then there exists a unique $T_{t}$-invariant normal conditional expectation $E$ of $\mathscr{M}$ onto the fixed point subalgebra $\mathscr{M}(T)$ given by

$$
E(A)=w^{*}-\lim _{\lambda \rightarrow 0} \lambda \int_{0}^{\infty} d t e^{-\lambda t} T_{t}(A), \quad A \in \mathscr{M},
$$

the integral being a weak* Riemann integral.

$\operatorname{Proof}(c f .[16])$. The net $\left\{\lambda \int_{0}^{\infty} d t e^{-\lambda t} T_{t}(A)\right\}_{\lambda}$ is bounded in norm by $\|A\|$, hence, by the weak ${ }^{*}$ compactness of the unit ball of $\mathscr{M}$, it converges in the weak ${ }^{*}$ topology as $\lambda \rightarrow 0$ if and only if it has exactly one weak* limit point. Let $A_{0}$ be one of such limit points. By [17], Theorems 18.6.2 and 18.7.3, $s-\lim _{\lambda \rightarrow 0} \lambda \int_{0}^{\infty} d t e^{-\lambda t} \hat{T}_{t}$ exists and is the projection $P$ onto the subspace of $\hat{T}_{t}$-invariant vectors in $\mathscr{H}$. Then $P A \Omega=$ $A_{0} \Omega=P A_{0} \Omega$. This proves that $A_{0}$ is uniquely determined by $A$ and is in $\mathscr{M}(T)$, since $\Omega$ is separating for $\mathscr{M}$. Let $A_{0}=E(A)$. It is clear that $E$ is linear and completely positive, and that $E(A)=A$ if and only if $A$ is in $\mathscr{M}(T)$. If $A \in \mathscr{M}, B_{1}, B_{2} \in \mathscr{M}(T)$, then $E\left(B_{1} A B_{2}\right)=B_{1} E(A) B_{2}$, since $T_{t}\left(B_{1} A B_{2}\right)=B_{1} T_{t}(A) B_{2}$ for all $t$, by [7], Theorem 3.1. Hence $E$ is a conditional expectation, which is normal since it has a predual map $E_{*}$, whose explicit expression on the dense set $\mathscr{V}$ is

$$
E_{*} \psi(A)=(P \Psi, A \Omega) \text { if } \psi(A)=(\Psi, A \Omega) .
$$

Moreover, $E$ is $T_{t}$-invariant by construction. If $E^{\prime}$ is another $T_{t}$-invariant normal conditional expectation of $\mathscr{M}$ onto $\mathscr{M}(T)$, then $E^{\prime}=E E^{\prime}=E^{\prime} E=E$. 
Corollary 2.2. A normal functional $\varphi$ on $\mathscr{M}$ is $T_{t}$-invariant if and only if it is of the form

$$
\varphi=(\varphi \uparrow \mathscr{M}(T)) \circ E .
$$

In particular, $\omega=(\omega) \cdot \mathscr{M}(T)) \circ E$, and $E$ is faithful since $\omega$ is.

By Corollary 2.2, the study of normal $T_{t}$-invariant states on $\mathscr{M}$ is reduced to the study of normal states on $\mathscr{M}(T)$. The fact that $\mathscr{M}(T)$ is the range of a faithful normal conditional expectation $E$ with $\omega \circ E=\omega$ gives restrictions on $\mathscr{M}(T)$, and hence on the set of $T_{t}$-invariant states in $\mathscr{M}_{*}$. Indeed, by [18], $\mathscr{M}(T)$ is globally invariant under the modular automorphism group $\sigma_{t}$ associated to $\omega$ by TomitaTakesaki theory [19], and if $\mathscr{M}$ is a type I factor, then $\mathscr{M}(T)$ is of type I and its centre is totally atomic [20].

It is clear that $\omega$ is the unique stationary state for $T_{t}$ in $\mathscr{M}_{*}$ if and only if $\mathscr{M}(T)=$ $\mathbb{C} \mathbb{1}$ (cf. $[3,4]$ ). In the more general situation, we have the following

Proposition 2.3. A state $\varphi$ in $\mathscr{M}_{*}$ is $T_{t}$-invariant and majorized by a scalar multiple of $\omega$ if and only if it is of the form

$$
\varphi(A)=\omega(B)^{-1}(J B \Omega, A \Omega), A \in \mathscr{M},
$$

for some positive $B$ in $\mathscr{M}(T), J$ being the antiunitary involution on $\mathscr{H}$ such that $J \Omega=\Omega, J \mathscr{M} J=\mathscr{M}^{\prime}$.

Proof. The general form of such a state $\varphi$ is

$$
\varphi(A)=(\Omega, X \Omega)^{-1}(X \Omega, A \Omega)
$$

for some positive $X$ in $\mathscr{M}^{\prime}$, with $X \Omega$ in $P \mathscr{H}$ (see e.g. [21], Proposition 2.5.I). Then $B=J X J$ is a positive element of $\mathscr{M}$, and $B \Omega=J X \Omega$ is in $P \mathscr{H}$, since $\mathscr{M}(T)$ is stable under the modular automorphism group and $P \mathscr{H}=\overline{\mathscr{M}(T) \Omega}$. Hence $B$ is in $\mathscr{M}(T)$ since $\Omega$ is separating for $\mathscr{M}$, and $\varphi$ can be written in the form (2.2).

Remark. The state $\varphi$ of the above Proposition is extremal $T_{t}$-invariant if and only if $B$ is a minimal projection in $\mathscr{M}(T)$.

An adaptation of the arguments of [22], Theorem 6.4.1 gives the following

Proposition 2.4. If $\mathscr{M}(T)$ is abelian, there exists a unique maximal measure $\mu$ on the set of $T_{t}$-invariant states on $\mathscr{M}$, with resultant $\omega$ and such that

$$
\int \prod_{i=1}^{n} \varphi\left(A_{i}\right) d \mu(\varphi)=\omega\left(\prod_{i=1}^{n} E\left(A_{i}\right)\right)
$$

for all self-adjoint $A_{i}$ in $\mathscr{M}, i=1, \ldots, n$, and for all $n$.

\section{Weak Approach to Equilibrium}

In this Section, we discuss a condition under which any state $\psi$ in $\mathscr{M}_{*}$, acted upon by $\left(T_{t}\right)_{*}$, converges weakly as $t \rightarrow \infty$ to a limit, which is given by $(\psi+\mathscr{U}(T))_{\circ} E$.

Definition. The integrated form of the dissipation function $D_{t}(.,$.$) is defined on$ $\mathscr{M} \times \mathscr{M}$, with values in $\mathscr{M}$, by 


$$
D_{t}(A, B)=T_{t}\left(A^{*} B\right)-T_{t}\left(A^{*}\right) T_{t}(B) .
$$

$D_{t}(A, A) \geqq 0$ for all $A$ in $\mathscr{M}, t \geqq 0$ by the Kadison-Schwarz inequality [22]. Denote by $\mathcal{N}(T)$ the null space of $\left\{D_{t}: t \geqq 0\right\}$, i.e.

$$
\mathscr{N}(T)=\left\{A \in \mathscr{M} ; D_{t}(A, A)=0 \text { for all } t \geqq 0\right\} .
$$

In general, $\mathscr{N}(T)$ contains $\mathscr{M}(T)$.

Theorem 3.1. Let $T_{t}$ be a dynamical semigroup of a von Neumann algebra $\mathscr{M}$, with a faithful normal stationary state $\omega$. If $\mathscr{N}(T)=\mathscr{M}(T)$, then

$$
w^{*}-\lim _{t \rightarrow \infty} T_{t}(A)=E(A) \text { for all } A \text { in } \mathscr{M} .
$$

Proof. For all $A, B$ in $\mathscr{M}$, we have

$$
\begin{aligned}
\lim _{t \rightarrow \infty} \omega\left(D_{t}(A, B)\right) & =\lim _{t \rightarrow \infty}\left(A \Omega,\left[\mathbb{1}-\hat{T}_{t}^{*} \hat{T}_{t}\right] B \Omega\right) \\
& =\left(A \Omega,\left[\mathbb{1}-Q^{2}\right] B \Omega\right),
\end{aligned}
$$

where $Q^{2}=s-\lim _{t \rightarrow \infty} \hat{T}_{t}^{*} \hat{T}_{t}([23]$, p. 41). Then the expression

$$
\begin{aligned}
\omega\left(D_{t}\left(T_{s}(A), T_{s}(A)\right)\right) & =\left(A \Omega,\left[\hat{T}_{s}^{*} \hat{T}_{s}-\hat{T}_{t+s}^{*} \hat{T}_{t+s}\right] A \Omega\right) \\
& =\omega\left(D_{t+s}(A, A)\right)-\omega\left(D_{s}(A, A)\right)
\end{aligned}
$$

tends to zero as $s \rightarrow \infty$, for all $t$ and for all $A$. Hence, using the Schwarz inequality for the positive sesquilinear form $\omega\left(D_{t}(.,).\right)$, we find that $\omega\left(D_{t}\left(T_{s}(A), B\right)\right)$ tends to zero as $s \rightarrow \infty$, for all $t \geqq 0$ and for all $A, B$ in $\mathscr{M}$. This proves that any weak* limit point $A_{\infty}$ as $s \rightarrow \infty$ of the norm-bounded net $\left\{T_{s}(A)\right\}_{s}$ is in $\mathcal{N}(T)$.

Since $E$ is a normal $T_{t}$-invariant conditional expectation, $T_{s}(A)=E(A)+$ $(I-E) T_{s}(A)$ for all $s$, and $A_{\infty}=E(A)+(I-E) A_{\infty}$. If $\mathscr{N}(T)=\mathscr{M}(T), \quad(I-$ E) $A_{\infty}=0$, and $A_{\infty}=E(A)$. This holds for any weak* limit point, hence (3.3) follows from the weak* compactness of the unit ball of $\mathscr{M}$.

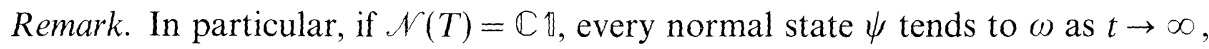
under the action of $\left(T_{t}\right)_{*}$. The same result has been derived by Albeverio and H $\phi$ egh-Krohn [4] under the (less restrictive) assumptions that $\mathscr{M}(T)=\mathbb{C} \mathbb{1}$ and $T_{t}$ has no (proper) eigenvalue on the unit circle besides 1 . Although less powerful from the mathematical point of view, our result is perhaps easier to use in applications to the approach to stationarity of open quantum systems, moreover, we are able to study situations when the stationary state is not unique, such as the quantum measurement process.

For the rest of this Section, we consider the case $\mathscr{M}=\mathscr{B}\left(\mathscr{H}^{0}\right)$, the algebra of all bounded operators on a separable Hilbert space $\mathscr{H}^{0}$, and $T_{t}=\exp L t, L$ being a bounded linear map of $\mathscr{B}\left(\mathscr{H}^{0}\right)$ into itself. The general form of $L$, given in [13] is

$$
L(A)=\sum_{i} V_{i}^{*} A V_{i}+K^{*} A+A K,
$$

where $K, V_{i}$ are in $\mathscr{B}\left(\mathscr{H}^{0}\right), \sum_{i} V_{i}^{*} V_{i}+K^{*}+K=0$, and the series converge ultraweakly. If $A$ is in $\mathscr{N}(T)$, then 


$$
\dot{D}(A, A) \equiv L\left(A^{*} A\right)-L\left(A^{*}\right) A-A^{*} L(A)=\left.\frac{d}{d t} D_{t}(A, A)\right|_{t=0}=0 .
$$

Thus $\mathscr{N}(T) \subset \operatorname{Ker} \dot{D}$. When $L$ has the form (3.4), Ker $\dot{D}=\left\{V_{i}\right\}^{\prime}$, the commutant being taken in $\mathscr{B}\left(\mathscr{H}^{0}\right)[2,7]$.

We have the following

Theorem 3.2. Let $T_{t}=\exp$ Lt be a dynamical semigroup of $\mathscr{B}\left(\mathscr{H}^{0}\right)$, with Lof the form (3.4). Assume that $\operatorname{lin}\left\{V_{i}\right\}$ is a self-adjoint set, and $\left\{V_{i}\right\}^{\prime}=\mathbb{C} \mathbb{0}$. Then, if $T_{t}$ has a normal stationary state $\omega, \omega$ is faithful and $w^{*}-\lim _{t \rightarrow \infty} T_{t}(A)=\omega(A) \mathbb{1}$ for all $A$ in $\mathscr{B}\left(\mathscr{H}^{0}\right)$.

Proof. If $\operatorname{lin}\left\{V_{i}\right\}$ is self-adjoint, $\left\{V_{i}\right\}^{\prime}=\mathbb{C} \mathbb{Q}$ is equivalent to "there is no proper closed subspace of $\mathscr{H}^{0}$ which is stable under all $V_{i}$ 's" (see e.g. [21], 2.2.4 and 2.3.I). Then $T_{t}$ is irreducible in the sense of Davies and $\omega$ is faithful [5]. Now Theorem 3.1

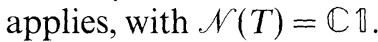

Remark. Notice that, in contrast to the corresponding theorems for the case of a finite-dimensional $\mathscr{H}^{0}[2,5]$, the existence of a normal stationary state must be assumed in advance. Counterexamples when no stationary state exists are discussed in $[5,7]$.

Theorem 3.1 can be applied to the study of the reduced dynamics of a quantum system weakly coupled to several heat reservoirs at nonzero temperatures, for in that case $L$ is known and $\operatorname{lin}\left\{V_{i}\right\}$ is a self-adjoint set $[2,8]$.

Assume now that $T_{t}$ has a faithful stationary state which is not unique. It follows from the discussion of Section 2 that there exists a family $\left\{P_{n}\right\}$ of mutually orthogonal $T_{t}$-invariant projections in $\mathscr{B}\left(\mathscr{H}^{0}\right)$, with $\sum_{n} P_{n}=1$, such that the centre $\mathscr{Z}$ of $\mathscr{M}(T)$ is $\left\{P_{n}\right\}^{\prime \prime}$. We consider two cases:

$$
\begin{array}{ll}
\text { (i) } \mathscr{M}(T)=\mathscr{Z} \text { : then } E(A)=\sum_{n} \omega\left(P_{n}\right)^{-1} \omega\left(P_{n} A P_{n}\right) P_{n} & \text { (cf. [24]); } \\
\text { (ii) } \mathscr{M}(T)=\mathscr{Z}^{\prime}: \text { then } E(A)=\sum_{n} P_{n} A P_{n} & \text { (cf. [6], p. 59). }
\end{array}
$$

Indeed, it is easily checked that the maps defined above are $T_{t}$-invariant normal conditional expectations onto $\mathscr{Z}$ (resp. $\mathscr{Z}^{\prime}$ ), by using the fact that $P_{n} T_{t}(A) P_{n}=$ $T_{t}\left(P_{n} A P_{n}\right)$ by [7] Theorem 3.1. The two maps are possible choices for the "reduction of the wave-packet" occurring when an observable $B=\sum_{n} b_{n} P_{n}$ is measured.

When all $P_{n}$ 's are one-dimensional, the cases (i) and (ii) coincide, otherwise, one usually chooses (ii) on the basis of a hypothesis of minimum disturbance. However, the analysis of Accardi [25] should lead to prefer (i). The condition $\mathscr{N}(T)=\mathscr{M}(T)$ is satisfied when

$$
\left\{V_{i}\right\}^{\prime}=\left\{V_{i}, V_{i}^{*}, K, K^{*}\right\}^{\prime} \quad(\text { see }[7]) .
$$

In this situation, the asymptotic effect of $T_{t}$ is the measurement of $B$. If one requires minimum disturbance, then $\left\{V_{i}\right\}^{\prime}=\left\{P_{n}\right\}^{\prime}$, and $V_{i}=\sum_{n} c_{i n} P_{n}$. This proves that the model of [9] is essentially unique. 
Finally, we remark that, if $\omega$ is not assumed to be faithful, the proof of Theorem 3.1 can be adapted to show that, if $\omega\left(D_{t}(A, A)\right)=0$ for all $t$ implies $A \Omega$ to be a multiple of $\Omega$, then $\lim _{t \rightarrow \infty} \omega\left(A T_{t}(B)\right)=\omega(A) \omega(B)$ for all $A, B$ in $\mathscr{M}$.

\section{Strong Approach to Equilibrium}

For an infinitely extended quantum system, a property of approach to equilibrium like (3.3) can hold even if $T_{t}$ is a group of automorphisms of $\mathscr{M}$. A possible characterization of true dissipation of local disturbances is [10]

$$
\lim _{t \rightarrow \infty}\left\|\psi \circ T_{t}-\psi \circ E\right\|=0 \text { for all } \psi \text { in } \mathscr{M}_{*} .
$$

Lemma 4.1. A sufficient condition for (4.1) to hold is

$$
s-\lim _{t \rightarrow \infty} \hat{T}_{t}^{*} \uparrow(1-P) \mathscr{H}=0,
$$

where $P$ is the projection onto the subspace of $\hat{T}_{t}$-invariant vectors in $\mathscr{H}$.

Proof. Let $\psi$ be in $\mathscr{V}, \psi(A)=(\Psi, A \Omega), \Psi \in \mathscr{H}$. Then

$$
\begin{aligned}
& \left|\psi\left(T_{t}(A)\right)-\psi(E(A))\right|=\left|\left(\Psi, \hat{T}_{t} A \Omega\right)-(\Psi, P A \Omega)\right| \\
& \quad=\left|\left(\hat{T}_{t}^{*}(\mathbb{1}-P) \Psi, A \Omega\right)\right| \leqq\left\|\hat{T}_{t}^{*}(\mathbb{1}-P) \Psi\right\| \cdot\|A\|,
\end{aligned}
$$

which tends to zero as $t \rightarrow \infty$, uniformly in $A \in \mathscr{M}$, if (4.2) holds. Since $\mathscr{V}$ is uniformly dense in $\mathscr{M}_{*}$, (4.1) follows.

Conditions ensuring the validity of (4.2) are discussed in [23] Chapter II, Theorem 2.1 and Proposition 6.7; however, they are not suitable for applications in concrete cases. We are able to prove the property (4.1) under the assumption $\mathscr{N}(T)=\mathscr{M}(T)$ for the particular class of dynamical semigroups defined below.

Definition. A dynamical semigroup $T_{t}$ of a von Neumann algebra $\mathscr{M}$ will be said to be normal w.r.t, a faithful normal state $\omega$ if there exists another dynamical semigroup $T_{t}^{+}$of $\mathscr{M}$ such that

$$
\omega\left(T_{t}^{+}(A) B\right)=\omega\left(A T_{t}(B)\right) \text { for all } A, B \text { in } \mathscr{M}, t \geqq 0
$$

and

$$
T_{t} T_{s}^{+}=T_{s}^{+} T_{t} \text { for all } t, s \geqq 0 .
$$

Remark. In particular, $\omega$ is stationary under $T_{t}$ and $T_{t}^{+}$. Conditions (4.3) and (4.4) hold for instance when $T_{t}$ satisfies detailed balance w.r.t. $\omega$ in the sense of [26] and the Hamiltonian part of $T_{t}$ is the modular automorphism group associated to $\omega$ (cf. also [27]); for the reduced dynamics of a spatially confined quantum system in the weak coupling limit [28] this situation occurs when the energy level shift is proportional to the original Hamiltonian.

Theorem 4.2. For a dynamical semigroup $T_{t}$ which is normal w.r.t. $\omega$, the following are equivalent

(i) $\mathscr{N}(T)=\mathscr{M}(T)$;

(ii) for all $\psi$ in $\mathscr{M}_{*}, \psi \circ T_{t}$ converges in the norm topology of $\mathscr{M}_{*}$ as $t \rightarrow \infty$; 
and if the above equivalent conditions hold, the limit of $\psi \circ T_{t}$ as $t \rightarrow \infty$ is $\psi \circ E$.

Proof. Denote by $Q$ the positive square root of $s-\lim _{t \rightarrow \infty} \hat{T}_{t}^{*} \hat{T}_{t}([23], \mathrm{p}$. 41). It follows from (4.4) that $\left\{\hat{T}_{t}, \hat{T}_{t}^{*}\right\}$ is an abelian family of operators, hence $Q$ is a projection commuting with $\hat{T}_{t}^{*}$ and $\hat{T}_{t}$, and also $s-\lim _{t \rightarrow \infty} \hat{T}_{t} \hat{T}_{t}^{*}=Q$. Then $Q \mathscr{H}$ reduces both $\hat{T}_{t}$ and $\hat{T}_{t}^{*}$, and by $\left([23]\right.$ p. 40-41) the restrictions of $\hat{T}_{t}$ and $\hat{T}_{t}^{*}$ to $(1-Q) \mathscr{H}$ contract strongly to zero as $t \rightarrow \infty$, whereas their restrictions to $Q \mathscr{H}$ are isometric. Now $Q$ maps $\mathscr{M} \Omega$ into $\mathscr{M} \Omega$ (just let $A_{\infty}$ be a weak* limit point as $t \rightarrow \infty$ of the norm bounded net $\left\{T_{t}^{+} T_{t}(A)\right\}_{t}$, then $\left.Q A \Omega=A_{\infty} \Omega\right)$ and $\|(\mathbb{Z}-Q) A \Omega\|^{2}=\lim _{t \rightarrow \infty} \omega\left(D_{t}(A, A)\right)$ for all $A$ in $\mathscr{M}$; hence $Q \mathscr{H}$ is the closure of $\mathscr{N}(T) \Omega$. It follows that $\mathscr{N}(T)$ is globally invariant under $T_{t}$ and $T_{t}^{+}$, and

$$
T_{t}^{+} T_{t} \uparrow \mathscr{N}(T)=T_{t} T_{t}^{+} \uparrow \mathcal{N}(T)=I \uparrow \mathscr{N}(T) .
$$

(i) $\Rightarrow$ (ii) :(i) implies that $P=Q$, hence Lemma 4.1 can be applied and (ii) follows, with $\lim _{t \rightarrow \infty} \psi \circ T_{t}=\psi \circ E$.

(ii) $\Rightarrow$ (i) : Let $\psi$ be in $\mathscr{M}_{*}$, and take $\varphi$ in $\mathscr{M}_{*}$ such that $\left\|\psi \circ T_{t}-\varphi\right\| \rightarrow 0$ as $t \rightarrow \infty$. Then $\varphi$ is $T_{t}$-invariant. Denote by $\hat{\psi}, \hat{\varphi}$ the restrictions of $\psi, \varphi$ to $\mathcal{N}(T)$. Taking into account Equation (4.5) we have

$$
\left\|\psi \circ T_{t}-\varphi\right\|=\left\|(\psi-\varphi) \circ T_{t}\right\| \geqq\left\|(\hat{\psi}-\hat{\varphi}) \circ T_{t}\right\|=\|\hat{\psi}-\hat{\varphi}\| .
$$

Then, for any $\psi$ in $\mathscr{M}_{*}$, there exists $\varphi=\varphi(\psi)$ in $\mathscr{M}_{*}$ such that

$$
\hat{\psi}=\hat{\varphi}=[(\varphi \uparrow \cdot \mathscr{M}(T)) \circ E] \uparrow \cdot \mathcal{N}(T) .
$$

This can only hold if $\mathscr{M}(T)=\mathscr{N}(T)$, which proves (i).

Acknowledgements. I gratefully acknowledge stimulating discussions and correspondence with E. B. Davies and D. E. Evans. I am also grateful to G. M. Prosperi fot the suggestion of studying the problem when the stationary state is not unique. I thank V. Gorini, G. Parravicini and M. Verri for some discussions.

\section{References}

1. Spohn, H. : Rept. Math. Phys. 10, 189-194 (1976)

2. Spohn, H. : Lett. Math. Phys. 2, 33-38 (1977)

3. Frigerio, A. : Lett. Math. Phys. 2, 79-87 (1977)

4. Albeverio, S., Hфegh-Krohn, R. : Frobenius theory for positive maps of von Neumann algebras. Preprint, Oslo (1977)

5. Davies, E. B. : Commun. math. Phys. 19, 83-105 (1970)

6. Davies, E. B. : Quantum theory of open systems. London: Academic Press 1976

7. Evans, D. E. : Commun. math. Phys. 54, 293-297 (1977)

8. Spohn, H., Lebowitz, J. : Irreversible thermodynamics for quantum systems weakly coupled to thermal reservoirs. Advan. Phys. Chem. (to appear)

9. Ghirardi, G. C., Rimini, A., Weber, T. : Nuovo Cimento 30B, 133-144 (1975)

10. Davies, E. B. : Commun. math. Phys. 55, 231-258 (1977)

11. Ingarden, R. S., Kossakowski, A. : Ann. Phys. (NY) 89, 451-485 (1975)

12. Gorini, V., Kossakowski, A., Sudarshan, E. C. G. : J. Math. Phys. 17, 821-825 (1976)

13. Lindblad, G. : Commun. math. Phys. 48, 119 130 (1976) 
14. Evans, D. E., Lewis, J. T. : J. Funct. Anal. 26, 369-377 (1977)

15. Radin, C. : Commun. math. Phys. 21, 291-302 (1971)

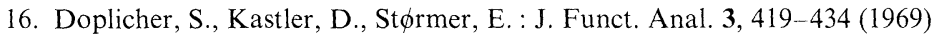

17. Hille, E., Phillips, R. : Functional analysis and semi-groups. Providence: American Mathematical Society 1957

18. Takesaki, M. : J. Funct. Anal. 9, 306-321 (1972)

19. Takesaki, M. : Tomita's theory of modular Hilbert algebras and its applications. Berlin, Heidelberg, New York: Springer 1970

20. St $\phi$ rmer, E. : Math. Scand. 30, 46-50 (1972)

21. Dixmier, J. : Les $C^{*}$-algèbres et leurs représentations. Paris: Gauthier-Villars 1969

22. Kadison, R. V. : Ann. Math. 56, 494-503 (1952)

23. Sz.-Nagy, B., Foias, C. : Harmonic analysis of operators on Hilbert space. Amsterdam: North Holland 1970

24. Gudder, S., Marchand, J. P. : J. Math. Phys. 13, 799 806 (1972)

25. Accardi, L. : Advan. Math. (to appear)

26. Kossakowski, A., Frigerio, A., Gorini, V., Verri, M. : Commun. math. Phys. 57, $97-110$ (1977)

27. Alicki, R. : Rept. Math. Phys. 10, 249-258 (1976)

28. Davies, E. B. : Commun. math. Phys. 39, 91-110 (1974)

Communicated by H. Araki

Received April 10, 1978

\section{Note Added in Proof}

After the completion of this paper, I have been informed that Theorem 2.1 has been proved independently by S. Watanabe (Niigata University, Niigata, Japan). 